\title{
REMARQUES SUR LA TECHNIQUE MYCOLOGIQUE (1)
}

\author{
Par Luz Maria DALMAU
}

Un des plus grands obstacles à la détermination précise des champignons pathogènes est le manque d'une technique type qui permette de mettre sûrement en évidence leurs particularités caractéristiques. Le but de ce travail est précisément d'aider le débutant à reconnaître les champignons qu'on rencontre couramment dans les recherches de mycologie médicale.

1. Méthode d'isolement en culture pure en partant des lésions de la peau. - Nettoyer soigneusement la lésion à l'alcool à $95^{\circ}$ puis à l'éther. Gratter avec un scalpel stérile bien affilé et à tranchant bombé ou raser avec un rasoir de sûreté stérilisé, jusqu'à ce que la sérosité sanguinolente commence à sourdre. Faire tomber dans une boîte de Petri stérile les squames ainsi détachées de la peau ou restées adhérentes à la lame du scalpel ou du rasoir. Ne pas oublier de noter le nom du malade, la date du prélèvement et-les renseignements cliniques. Si possihle, compléter ces indications par un dessin ou une photographie.

En arrivant au laboratoire et après avoir prélevé les fragments nécessaires à l'examen microscopique direct (par macération dans la potasse à $400 / 0$ ), verser dans la boîte de Petri renfermant les squames juste assez de gélose de Sabouraud glycosée ou maltosée pour former sur le fond une mince couche, dans laquelle les squames se trouvent enrobées. Pour réduire les chances de contamination bactérienne, on peut au préalable traiter les squames par l'alcool, mais cette précaution nous paraît inutile car elle ne diminue pas la contamination par les champignons saprophytes vulgaires et peut empêcher le développement du champignon pathogène.

Lorsque les colonies commencent à se développer et à être perceptibles à l'œil nu, il faut, avec le crayon gras, les entourer d'un cercle et les numéroter, puis les transplanter sur.milieu d'épreuve de Sabouraud en tubes inclinés et aussi sur milieu de conservation non sucré. Ce repiquage est nécessaire pour empêcher les jeunes colonies d'être étouffées dans la suite par le développement luxu-

(1) Traduit de l'anglais par le Dr Maurice Langeron.

Annales de Parasitologie, $\mathrm{T}$. VII, $\mathrm{N}^{\circ} 6 .-1^{\mathrm{er}}$ novembre 1929 , p. 536-545. 
riant des contaminations, telles que les Penicillium et les Aspergillus.

Cette opération exige certaines précautions pour obtenir des cultures pures. Si la colonie est molle, il suffit de la toucher avec l'aiguille métallique flambée, d'émulsionner la prise dans quelques $\mathrm{cm}^{3}$ d'un liquide nutritif quelconque, d'ensemencer avec cette émulsion des tubes de milieu de Sabouraud, puis de verser les cultures dans des boîtes de Petri par la méthode des trois plaques de Koch. En pratique, cependant, deux plaques suffisent.

Si la colonie est dure, tenace, cartonnée, il faut en détacher une parcelle avec une aiguille hypodermique en platine iridié montée sur une baguette de verre. Cette parcelle sera coupée, écrasée et dissociée contre la paroi d'un tube garni d'un liquide nutritif et les fragments seront incorporés à ce liquide. On laisse macérer pendant un jour environ, en agitant de temps en temps pour favoriser la désarticulation du mycélium. Lorsque le développement a commencé dans ces conditions, on agite et on prépare comme plus haut des plaques de milieu de Sabouraud.

Lorsqu'un organisme intéressant persiste à vivre en symbiose avec un saprophyte banal, champignon ou bactérie, on peut encore arriver à le débarrasser de cette contamination. Le premier moyen et peut-être le plus efficace est d'augmenter l'acidité du milieu ; alors les bactéries et quelques champignons cessent de prospérer tandis que l'organisme pathogène peut continuer à vivre. On peut aussi essayer le chauffage à $50^{\circ}$ et finalement avoir recours aux produits chimiques et aux colorants. Lorsque des colonies paraissant intéressantes se montrent sur ces plaques, on les pêche et on les transporte sur milieu d'épreuve incliné. Généralement, les cultures ainsi obtenues sont pures.

2. Isolement des champignons des selles, du grattage de la langue, etc. - Pratiquement, on a recours à la méthode d'Anderson. On laisse d'abord les plaques se solidifier, puis on les touche 25 fois avec l'anse rechargée plusieurs fois avec le matériel à ensemencer. Après environ quatre jours, on pêche les colonies et on purifie les différentes espèces par la méthode de Koch. Par ce moyen, le pourcentage des points qui renferment des colonies semblables donne une idée approximative de l'abondance des germes dans le matériel.

3. Cultures en blocs suspendus (hanging block). - On prépare ces cultures avec les colonies pures obtenues sur les plaques. Nous préférons ce procédé à celui de la goutte pendante qui, pour les colonies très adhérentes, à mycélium radiculaire profond, présente 
des inconvénients évidents. En faisant le bloc, on doit couper dans la gélose deux jeunes colonies entières, avec l'aiguille métallique. Chacune est placée sur une lamelle stérilisée, dans une goutte de bouillon glycosé à 0,2 p. 100 , destinée à maintenir le bloc humide. Ces jeunes colonies peuvent être enlevées sans altérer notablement leur contour. Deux anneaux de verre de $15 \mathrm{~mm}$. de diamètre sur $3 \mathrm{~mm}$. de haut sont fixés sur une lame de $76 \times 51 \mathrm{~mm}$. On badigeonne le bord des anneaux avec de la vaseline stérilisée, de façon d'une part à les fixer sur la lame, d'autre part à fixer la lamelle. Le petit bloc de gélose se trouve ainsi suspendu dans une chambre humide scellée.

Bien que cette méthode du bloc suspendu soit considérée comme une des plus précises pour étudier les champignons, elle présente l'inconvénient de les enfermer dans une chambre close ne contenant qu'une quantité limitée d'oxygène ; lorsque celle-ci est consommée, un germe aérobie peut être arrêté dans son développement. Néanmoins, on peut obtenir ainsi de très belles préparations, de conservation indéfinie, en exposant ces cultures d'abord aux vapeurs d'acide osmique, puis aux vapeurs d'alcool et en montant dans le milieu de Farrant, dans une lame à concavité sur laquelle la lamelle est scellée avec un lut.

4. Cultures sur plaques. - L'examen des jeunes colonies sur plaques prend, dans notre laboratoire, une place de plus en plus importante dans la classification des champignons. Un des principaux avantages qu'elle présente sur la méthode des blocs suspendus est qu'on peut utiliser un des stades de la purification nécessaire de notre culture originale, sans avoir à effectuer le travail supplémentaire et délicat de la préparation du bloc suspendu. De plus, la colonie s'étale mieux dans la plaque et les hyphes fertiles sont plus rapidement découvertes et identifiées. Dans la plaque, il y a une aération suffisante, à travers les petites imperfections du bord et du couvercle; cela empêche l'arrêt de croissance des colonies. Il y a plus de chances de contamination par les spores provenant de l'air, mais si les plaques sont maniées avec soịn et tenues éloignées de l'haleine, on peut les conserver longtemps sans contamination. On peut objecter qu'en enlevant le couvercle pour examiner à un fort grossissement, la plaque sera sûrement contaminée, ce qui exclut tout examen ultérieur. Mais les colonies peuvent d'abord être examinées par la face inférieure de la boîte avec un objectif de $16 \mathrm{~mm}$. et on n'ouvre le couvercle que lorsque cet examen préliminaire a montré avec évidence des fructifications ou une disposition caractéristique des éléments. Alors la boite est 
ouverte et, en une séance, on examine, décrit et dessine avec les forts grossissements.

Le bloc suspendu est extrêmement utile pour suivre le développement de l'organisme à intervalles réguliers. La plaque paraît être plus pratique pour une détermination rapide. Avec l'objectif de $16 \mathrm{~mm}$., il est rarement possible d'être sûr du genre. C'est seulement en enlevant le couvercle et en employant l'objectif de $8 \mathrm{~mm}$. que les détails nécessaires deviennent apparents. En exposant quelque temps la colonie aux vapeurs de formol et en la recouvrant d'une goutte. d'huile de cèdre, il est possible de l'examiner avec l'objectif à immersion et de faire des mensurations exactes qui viennent s'ajouter à une plus grande richesse de détails. On peut même interposer une lamelle et la colonie peut souvent être étudiée avec succès de la manière ordinaire avec l'objectif à immersion.

La taille, la forme, la topographie superficielle et la consistance d'une colonie sur plaque ne sont pas toujours suffisantes pour la détermination. Si une colonie pure n'est pas bien diluée au début, quand on prépare la plaque, les colonies peuvent être petites, se toucher et ne pas présenter leurs détails caractéristiques. Un champignon pourvu d'un mycélium produit souvent des colonies parfaitement rondes, formées uniquement d'éléments levuriformes, dans la même plaque où on trouve d'autres colonies abondamment frangées d'hyphes ramifiées. Ces dernières s'observent surtout dans les cultures en souffrance qui sont souvent profondément enfoncées dans la gélose et se présentent sous la forme d'ovales, de croix, de triangles, etc., pourvus de franges. Dans la même plaque, quelques colonies peuvent être ternes et mates, tandis que les autres sont brillantes. Il est très fréquent de trouver un fort pourcentage de colonies poudreuses avec un petit nombre de colonies lisses, brillantes et plissées. Il n'est pas rare de rencontrer certaines colonies qui s'étendent en anneaux concentriques alors que d'autres ne s'étalent pas. Dans deux cas, il nous est arrivé de repiquer sur milieu de Sabouraud incliné 15 colonies différant les unes des autres comme nous venons de l'expliquer ; après développement, ces repiquages étaient macroscopiquement identiques.

Sur les plaques dont le contenu a été bien dilué au début et dont les colonies sont peu nombreuses et bien séparées, l'abondance de nourriture et la liberté du développément permettent à ce dernier d'être caractéristique.

Une autre considération importante dans l'emploi des cultures sur plaques, pour mettre en évidence la morphologie normale des colonies, est la facilité avec laquelle on peut les dessiner. Les croquis exécutés d'après une goutte de culture en bouillon, montée entre 
lame et lamelle, ne peuvent pas montrer les relations normales qui existent entre les éléments des champignons. Les photographies des colonies sur plaques sont plus difficiles à exécuter parce qu'il est impossible de mettre au point dans le mème plan un grand nombre d'éléments (voir pl. IX).

5. Comparaison entre le milieu d'épreuve et le milieu de conservation de Sabouraud. - Les milieux d'épreuve de Sabouraud renferment 4 p. 100 de glycose ou de maltose dans une gélose nutritive. L'addition de certains sucres, spécialement le glycose et le maltose, aux milieux gélosés, non seulement stimule la production des formes végétatives, mais fait ressortir la couleur et donne une croissance plus luxuriante. C'est un avantage important pour la différenciation précoce des champignons communs non pathogènes, tels que les Aspergillus, Penicillium, Sterigmatocystis, etc., d'autres champignons soupçonnés d'être la cause d'une maladie particulière.

Dans l'étude des dermatomycoses, les squames sont souvent semées sur milieu de conservation pour empêcher la formation d'un duvet pléomorphique. En effet, dès qu'un champignon se pléomorphise, ses véritables caractéristiques sont définitivement perdues et nè peuvent être récupérées ni par repiquage, ni par passage par les animaux. Lorsqu'ils sont devenus pléomorphiques, les champignons de certains genres, tels que les Trichophyton, Microsporum et Epidermophyton, ne montrent plus qu'un mycélium incolore avec de rares spores et leurs caractères génériques disparaissent. On peut dire, cependant, que beaucoup de champignons donnant des duvets, présentent souvent très clairement leurs particularités de reproduction dans la première culture sur milieu sucré. C'est seulement avec le temps et après des repiquages répétés sur milieux sucrés que ces organismes deviennent pléomorphiques.

Il est rarement possible de déterminer un organisme, même approximativement, seulement d'après l'aspect de son mycélium stérile. Des hyphes fertiles sont nécessaires. Par conséquent, pour tous les champignons qui ont tendance à devenir pléomorphiques et à se transformer en duvet, il est nécessaire de garder des souches sur milieu de conservation.

Ce milieu, qui, à cause de son manque de sucre, stimule la production des formes de résistance, spécialement des asques, trouve une autre application pour séparer les Saccharomyces des Torula et des Cryptococcus. Un des meilleurs moyens d'obtenir la formation des asques chez les Saccharomyces, est l'emploi du milieu de 
Gorodkowa qui consiste à ajouter 0,2 p. 100 de glycose ou de maltose à une gélose nutritive spéciale, pour faire partir la colonie ; la faible proportion de sucre est vite consommée et l'organisme est forcé par le jeûne à produire des ascospores. Jusqu'à ce qu'il ait été prouvé qu'une levure ne forme pas d'asques, il reste un doute sur sa classification parmi les Saccharomyces, chez lesquels ces milieux provoquent l'apparition des asques.

Les champignons intestinaux se développent très mal sur milieu de conservation, car ils paraissent exiger une quantité anormale de sucre.

\section{Caractères macroscopiques des cultures de champignońs. -} Le point important est d'avoir un milieu transparent pour l'observation du mycélium et des petites colonies sur plaques. L'aspect macroscopique d'une culture de champignon a une valeur considérable pour la classification, beaucoup plus qu'en bactériologie, pourvu que les milieux de culture soient universellement étalonnés et que les milieux employés au cours d'une recherche soient bien spécifiés. Des caractères importants, sont l'exubérance de la croissance, la couleur, la fluorescence, la transparence, la topographie de la surface : aplatie ou bombée, granuleuse, lisse, brillante, terne, en voile, sillonnée, rayonnée, vermiculaire, alvéolaire, piquetée, veloutée, sèche ou humide, de contour régulier ou non, circulaire, frangé ou nettement délimité, etc. L'observation de ces caractères macroscopiques commence sur la plaque et se continue sur les tubes inclinés et les cultures géantes.

7. Préparation des milieux de Sabouraud. - Nous croyons utile de donner intégralement notre technique, telle que nous la pratiquons dans notre laboratoire, plutôt que de supprimer des détails qu'un long usage nous a montrés utiles pour obtenir une gélose claire et stérile.

Il n'est pas économique de préparer à la fois moins de 5 à 6 litres donnant environ 600 tubes. Prendre trois fioles d'Erlenmeyer de deux litres et mettre dans chacune d'elles $40 \mathrm{gr}$. de gélose, $20 \mathrm{gr}$. de peptone et $1.500 \mathrm{~cm}^{3}$ d'eau. Chauffer pendant 30 minutes à $100^{\circ}$ dans un stérilisateur d'Arnold, jusqu'à dissolution de la gélose. On peut alors ajuster le $p \mathrm{H}$ avant le refroidissement, mais cette opération nous semble inutile parce que notre méthode nous fournit pratiquement un milieu de $p H$ constant $(6,5$ à 6,6$)$. Lorsqu'on désire connaître exactement le $p H$ ayant l'ensemencement, on le détermine sur un tube pris dans la fqurnée.

Lorsque le milieu est refroidi à $50^{\circ}$, on ajoute $75 \mathrm{gr}$. d'albumine d'œuf secouée dans un litre d'eau et on mélange bien. Les trois flacons sont alors placés dans le stérilisateur d'Arnold pendant une heure à $100^{\circ}$. Le coagulum ou gâteau qui se forme clarifie le milieu. On filtre sur coton, ou sur coton et mousseline. A ce moment, le volume du filtrat est 
mesuré (il y a toujours une perte par évaporation) et la quantité de sucre suffisante pour faire une solution à 4 p. 100 est dissoute dans le volume d'eau manquant et ajoutée au filtrat chaud.

On répartit alors en tubes et on stérilise à $100^{\circ}$ dans un stérilisateur d'Arnold, pendant 45 minutes, trois jours consécutifs. On peut alors incliner les tubes et les mettre à refroidir.

8. Cultures géantes. - L'avantage que présentent les cultures géantes est de mettre en évidence les particularités macroscopiques avec une vivacité qu'on ne peut obtenir sur plaques ou en tubes inclinés. Nous avons abandonné les fioles d'Erlenmeyer pour les boites de Kolle en verre pyrex, employées dans la fabrique de vaccin typhoïde-paratyphoïde de l'Ecole de médecine militaire. Ces cultures peuvent durer un an, sont faciles à manipuler sans danger de contamination ou de bris, économisent beaucoup d'espace, car on peut les superposer, et enfin sont très utiles pour l'enseignement et la photographie.

9. Cultures sur gélatine. - Dans la culture en piqûre, que nous employons couramment, les Torula et Cryptococcus donnent une ligne droite, sans expansions latérales ; c'est ce que nous appelons la culture en clou.

Les Saccharomyces donnent une culture en clou avec des nodosités latérales ou une fine expansion lichéniforme, presque imperceptible.

Monilia psilosis et Monilia krusei croissent abondamment et produisent des expansions fines, allongées en aiguille, partant de l'axe du clou et plus courtes vers son extrémité, ce qui donne à la culture la forme d'un pin renversé.

Monilia parapsilosis produit un aspect semblable, mais les expansions mycéliennes sont abondamment ramifiées latéralement en forme de sapin renversé.

Les Mycoderma intestinaux (nous avons, à la suite de Brumpt, exclu de la mycologie médicale le terme Oidium) liquéfient généralement la gélatine après avoir produit l'apparence de pin ou de sapin, lentement pour les uns, très rapidement pour d'autres.

10. Préparation de la gélatine. - A $900 \mathrm{~cm}^{3}$ de bouillon nutritif, ajouter $200 \mathrm{gr}$. de gélatine et chauffer au bain-marie jusqu'à dissolıtion. La bacto-gélatine et la marque Pfanstiehl sont très acides, au delà de 3 ou 6 à la phénolphtaléine. Ajuster la réaction à environ 1,5 ou tout autre $p H$ désiré. Laisser refroidir à $50^{\circ}$, ajouter les blancs de deux œufs secoués dans $100 \mathrm{~cm}^{3}$ de bouillon et porter rapidement à l'ébullition à $100^{\circ}$ pendant 10 minutes dans un double bouilleur, avec de la solution saturée de sel dans le compartiment inférieur. Le gâteau formé 
clarifie la solution. Filtrer au coton ou avec coton et gaze si c'est nécessaire. Répartir en tubes et stériliser à $100^{\circ}$, pendant 15 minutes, trois jours de suite. Refroidir rapidement en retirant du stérilisateur pour obtenir une gélatine dure, non liquéfiable à la température ordinaire ou dans la salle d'ensemencement à $28^{\circ}$.

11. Fermentation des sucres. - L'incorporation de 1 à $40 / 0$ de sucres à l'eau peptonée donne de bons renseigements sur les propriétés biochimiques des champignons. Au début, nous avons employé le bouillon comme dissolvant et support des sucres, mais il est plus simple de se servir d'eau peptonée. Pour l'essai des fermentations, il est très important d'opérer en milieu neutre et d'ajuster le $p \mathrm{H}$ à peu près à 7 . On note, à intervalles réguliers, les changements de réactions sous l'influence de la culture. Dans une longue série d'expériences, faites de 1914-1916, Ashford a décrit la formation de gaz et l'augmentation de l'acidité de certains milieux sous l'influence du Monilia psilosis et la réapparition de la neutralité dans l'espace d'environ deux semaines. Six sucres seulement ont donné cette réaction: glycose, lévulose, maltose, galactose, saccharose et dextrine, les trois premiers régulièrement, les trois autres très irrégulièrement. Tous les autres sucres n'ont donné aucune fermentation, mais en étendant ces investigations à d'autres champignons, il a choisi le maltose comme étalon de fermentation pour éliminer le Monilia psilosis qui seul, parmi tous les autres champignons intestinaux communs, à part certains Saccharomyces, fait fermenter ce sucre.

En étudiant d'autres champignons, il faut employer ces six sucres et en outre l'insuline, le raffinose et la mannite. L'arabinose, la dulcite, l'iso-dulcite et les sucres supérieurs plus rares et très coùteux fermentent rarement et leur emploi courant n'est pas nécessaire. Il y a très peu de champignons faisant fermenter le lactose, mais l'éclat et la variété morphologique des champignons dans les milieux préparés avec ce sucre et les sucres supérieurs, font qu'il vaut la peine de les y cultiver.

Les sucres facilement fermentescibles favorisent l'apparition des formes levures mais nuisent aux formes de résistance.

Toutefois, l'inconvénient de dépendre des épreuves de fermentation pour distinguer les espèces, notamment les Monilia, est que ces réactions ne sont pas constantes. Des espèces qui ont une ou deux fois fait fermenter certains sucres avec production de gaz, ont souvent dans la suite cessé de donner eetțe réaction et vice versa. Nous avons démontré que les rayons ultra-violets détruisent les pouvoirs fermentatifs du Monilia psilosis et que les rayons solaires agissent de même. 
Outre ces épreuves de fermentation, production de gaz et augmentation de l'acidité du milieu, il y a certains autres phénomènes importants dignes d'être notés dans les cultures en bouillon. La nature du dépôt est quelquefois un bon guide pour grouper les champignons; ce dépôt peut être granuleux et sablonneux, ou granuleux, ou filamenteux, ou muqueux. Les champignons troublent rarement les milieux liquides. Il y a des genres et des espèces qui forment une pellicule présentant des particularités caractéristiques ou une série de pellicules qui tombent l'une après l'autre au fond du tube, ou qui croissent en hauteur sous forme d'un duvet qui emplit le tube au-dessus du nivezit du liquide, ou qûi forment un collier en contact avec le sucre à la surface, ou enfin qui ne forment pas de pellicule. Il faut attendre au moins deux semaines avant de considérer la production de gaz comme négative. Nous avons trouvé très utiles les tubes en U de Dunham: on peut les placer dans des entailles coupées dans un bloc de bois qui peut porter ainsi 6 ou 8 tubes.

12. Lait tournesolé. - Pendant quelque temps nous avons employé ce milieu pour les champignons intestinaux, mais l'uniformité des résultats, c'est-à-dire l'alcalinité, apparaissant généralement après une très courte et douteuse période d'augmentation de l'acidité sans coagulation, dans le cas du Monilia psilosis, nous a engagé à abandonner son usage comme procédé courant ; mais il faut toujours l'employer lorsqu'on étudie une espèce nouvelle ou douteuse. Le lait de vache, qui arrive généralement avec une réaction acide, doit être légèrement alcalinisé avec de la soude $\mathrm{N} / 10$, puis partiellement privé de sa graisse, sédimenté et stérilisé, après addition d'une quantité suffisante d'azolitmine comme indicateur. On doit noter le changement de coloration, la coagulation et la peptonisation du caillot.

13. Coloration vitale. - Bien que cette méthode ne soit pas nouvelle, elle n'est pas aussi communément employée qu'elle devrait l'être pour l'étude des cultures en bouillon avec l'objectif

\section{Explication DE LA PLANCHE IX}

Exemples de colonies obtenues par la méthode de culture sur plaques exposée plus haut et photographiées directement avec l'objetif à immersion.

Fig. 1. - Mycoderma sp.

Fig. 2. - Sporotrichum beurmanni. 
ANNALES DE PARASITOLOGIE

T. VII, No 6, Novembre 1929.
PlaNChe IX.

(Mémoire Dalmad).

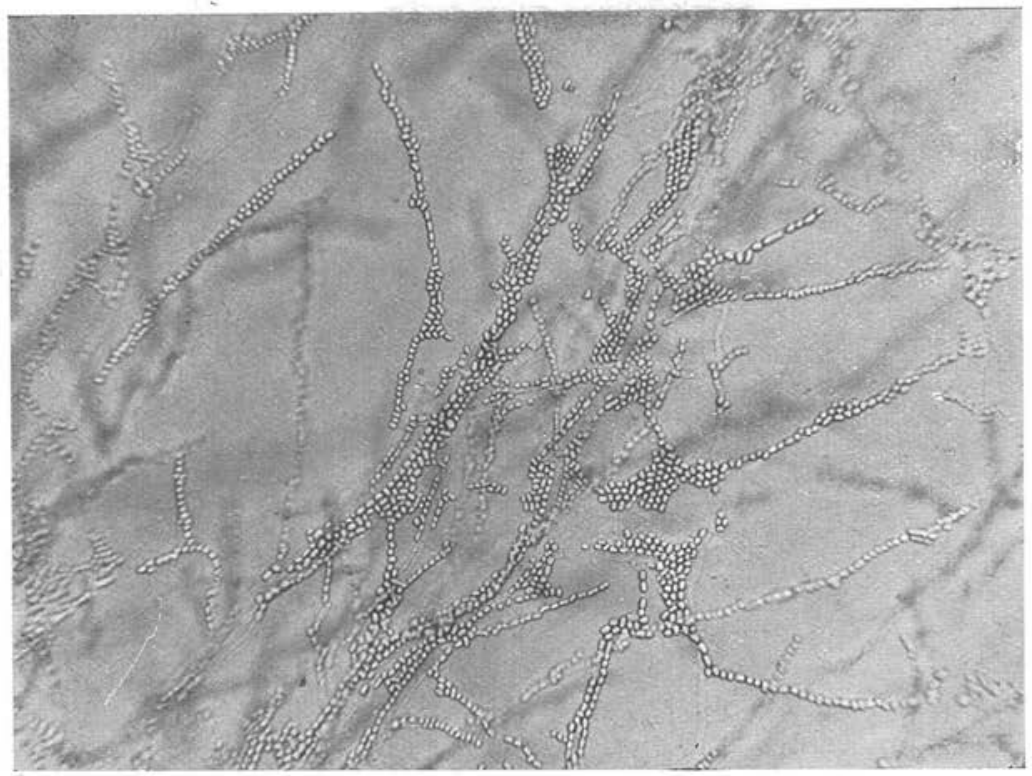

FIg. 1.

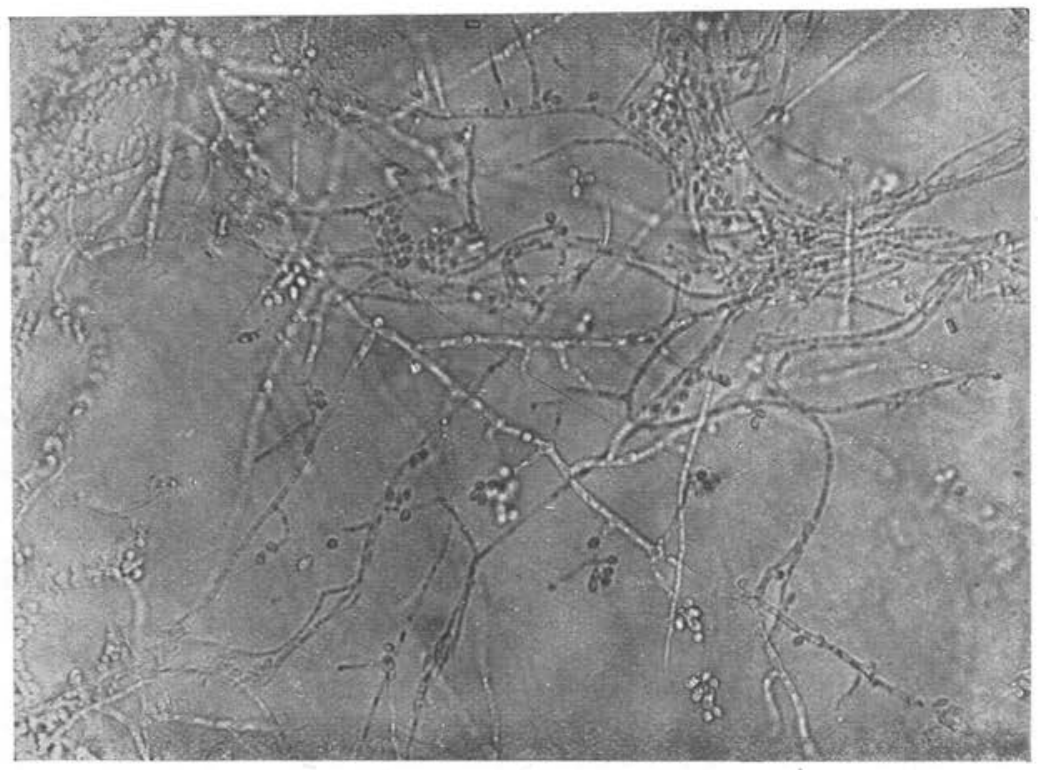

FIG. 2

Masson et Cie, Editeurs. 

à immersion. Sa valeur manifeste consiste à rendre bien plus visible la structure du thalle et des spores et à faire ressortir électivement la composition chimique des organes internes pendant que l'organisme est encore vivant.

Tout en,reconnaissant une certaine valeur à l'étude des champignons fixés et colorés, nous considérons en pratique qu'un organisme non coloré ou coloré vitalement, présente beaucoup plus d'intérêt, et nos conclusions concernant la morphologie sont en grande partie, et même presque exclusivement basées sur cet examen. L'étude d'une goutte de culture en bouillon est aussi un contrôle de la pureté.

La préparation des lames pour la coloration vitale est d'une importance capitale. Ces lames doivent être neuves, sans défauts et bien dégraissées par le mélange bichromate de potasse-acide sulfurique. Après neutralisation, il faut les essuyer avec un linge bien dégraissé. Avant usage, entever toute trace de poussière avec un pinceau neuf lavé à l'éther et séché. Déposer sur la lame une ou deux gouttes d'une solution d'un colorant vital (vert janus, rouge neutre, écarlate R.) à 1 p. 1.500 ou 1 p. 2.500 dans l'alcool éthylique. Laisser le colorant s'étendre sur toute la lame, ce qui aura lieu si celle-ci est parfaitement dégraissée. Laisser ensuite sécher à l'air. Une goutte de milieu liquide contenant le champignon est déposée sur une lamelle qu'on renverse sur la lame préalablement préparée avec le colorant. Laisser déposer et luter les bords de la lamelle avec de la vaseline. Examiner par intervalles. La coloration est plus intense lorsqu'on remplace le milieu liquide par une goutte de sérum sanguin clair dans laquelle on émulsionne un fragment de culture sur milieu solide.

On peut aussi employer les colorants habituels du sang (W'right, Leishman, Giemsa). Avec l'anse, on émulsionne un fragment d'une culture sur milieu de Sabouraud incliné, dans une goutte de sérum sanguin clair déposée à l'extrémité d'une lame. Avant dessiccation, on étale avec une autre lame, comme pour un frottis de sang. Fixer à l'alcool méthylique pendant une à trois minutes. Colorer comme pour le sang, en employant pour la dilution et le lavage, de l'eau bien neutre : la coloration doit durer de cinq à quinze minutes. Différencier pendant quelques secondes dans l'acide acétique à 1 p. 1000 et bien laver ensuite. L'addition de sérum empêche la contraction des éléments du champignon.

Ecole de médecine tropicale de l'Université de Porto-Rico.

AnNales de Parasitologie, T. VII, $\mathrm{N}^{\circ} 6 .-1^{\mathrm{er}}$ novembre 1929 .

35. 